Maurizio Cecconi

Manu L. N. G. Malbrain

\section{Cardiac output obtained by pulse pressure analysis: to calibrate or not to calibrate may not be the only question when used properly}

Received: 1 December 2012

Accepted: 2 December 2012

Published online: 4 January 2013

(C) Springer-Verlag Berlin Heidelberg and ESICM 2013

This editorial refers to the article available at: doi:10.1007/s00134-012-2786-7.

M. Cecconi (凶)

Critical Care Department, St George's Hospital,

London SW17 0QT, UK

e-mail: m.cecconi@nhs.net

M. L. N. G. Malbrain

Department of Intensive Care and High Care Burn Unit, Ziekenhuis Netwerk Antwerpen, ZNA Stuivenberg,

Lange Beeldekensstraat 267, 2060 Antwerp 6, Belgium

Cardiac output $(\mathrm{CO})$ is the main determinant of oxygen delivery and physical examination, and vital signs alone often fail to reflect significant derangements in CO [1]. Because of the complexity of assessment of clinical variables in unstable patients, direct measurement of $\mathrm{CO}$ is advisable. Pulse pressure (PP) analysis, often referred to as pulse contour (PC) analysis, is the analysis used by technologies that estimate changes in stroke volume (SV) and $\mathrm{CO}$ from an arterial pressure waveform signal $[2,3]$. The theory behind this approach dates back to the end of the nineteenth century when the German physiologist Otto Frank proposed the Windkessel (air chamber) model of cardiovascular physiology [4]. In a system with air and fluid, different air chambers were filled with air in order to simulate compliance, resistance and impedance of the cardiovascular system. This proved to be pivotal to our understanding of the importance of the relationship between flow and pressure. In the early twentieth century Erlanger and Hooker defined a relationship between stroke volume and arterial pressure, and crucially a correlation between SV and PP [5].
It was not until the development of modern computer technology that this theory could be put into practice and SV and CO tracked continuously on a beat to beat basis [6]. Since then the number of PP analysis devices has increased continuously over the years; some of these devices require an initial calibration; some are "self-calibrating" based on nomograms and anthropomorphic data $[7,8]$.

No matter which one of these technologies one wants to use, the understanding of the physiological principles behind it is the cornerstone for its proper use. Without this, monitors may be used inappropriately and patients put at risk.

So what are the important questions related to PP analysis? There are several and in this editorial we would like to focus mainly on four:

1. Are there any conditions in which PP analysis may suffer from intrinsic limitations?

2. What are the possible indications for PP analysis $\mathrm{CO}$ monitors?

3. How does calibration fit into this?

4. How to translate this into practice?

1. Pressure waves and flow waves are not the same thing. The algorithms behind PP analysis have to estimate flow from pressure. In those situations in which either the signal is poor (for instance severely underdamped or overdamped traces), or in which the relationship between pressure and flow is severely deranged, as in the case of severe aortic regurgitation or with counter-aortic balloon pumps, PP analysis will not provide reliable information [2].

2. PP analysis can be used to take a snapshot of the circulation or to look at how SV and CO change over time [9]. Reliability derives mainly from two principles: accuracy and trending ability. Understanding of accuracy (how close to the real absolute value a measurement is) and trending ability is very important in order to use the 
full potential of these devices [10]. Both these principles are used routinely at the bedside. For instance, when assessing a patient in shock, knowing if the $\mathrm{CO}$ is 2.0 or $15 \mathrm{~L} / \mathrm{min}$ may be important to confirm our diagnosis and plan and guide future management [11]. In this case we are looking at how accurate our estimation of $\mathrm{CO}$ is. When an accurate initial assessment has been performed (often with an intermittent dilution technique), it is often more crucial to know whether a clinical intervention is leading toward one direction or the other. As such, the absolute value of $\mathrm{CO}$ is less important than the trending ability of monitors to track changes continuously $[9,12]$. This is important because, for instance, it is unlikely that in patients with a hyperdynamic circulation (cirrhosis, pancreatitis, burns) PP analysis will provide accurate readings, unless calibrated frequently; however, trending over short periods of time may still be useful. There are also situations in which PP analysis simply does not represent a solution, because of limitations both in terms of accuracy and trending ability [13, 14]. Clinicians must be aware of these and that significant hemodynamic changes will affect the reliability of the devices in terms of accuracy and/or trending ability, as demonstrated in the present study.

3. Most PP analysis devices are a combination of two systems: a PP analysis algorithm and a calibration system. The PP analysis algorithm is the core of the monitor that allows beat to beat changes in SV and CO to be tracked. The calibration is what is used by the monitor to scale the estimated value of $\mathrm{CO}$ to an absolute value. While most calibrations will also provide additional information for the PP algorithm (therefore affecting also the way changes are detected) [15], their contribution relates more to the absolute value of $\mathrm{CO}$. When performing a calibration this has to be done properly: similarly to the zeroing of an arterial line, if the calibration process is not done properly it will add error, instead of increasing accuracy [16]. If done properly it can set or reset the continuous CO to an accurate value. This is important to keep in mind when assessing longer periods of trend, as most devices will show some drift over time or after significant changes in vascular impedance [17].

4. Most often PP analysis is used to assess the response to a fluid challenge. As such PP analysis can help us to solve therapeutic conflicts (defined as a situation where each of the possible therapeutic decisions carries some potential harm) [1, 18]. Therapeutic conflicts are the biggest challenge for protocolized hemodynamic management in critically ill patients. A therapeutic conflict is where our decisions can make the biggest difference. We have to recognize that $\mathrm{CO}$ measurement may be a lot less informative and accurate than we may want (or think). Continuity of CO measurement (and trending ability) offers vital insights that may be hidden in the analog signals of our monitors and may be more important than accuracy. "Physiological examination"-observing multiple parameters on the monitor in real time-should be considered to be (at least) as important as the classic "physical examination" $[1,19]$.

Most of the points we discussed have been explored in the study by Petzoldt et al. [20]. In this study they looked at the performance of calibrated and uncalibrated PC monitors in the context of severe valvular disease, comparing them to transoesophageal echocardiography. The calibrated SV ( $\mathrm{SV}_{\mathrm{PCCAL}}$ ) was measured via the $\mathrm{PiCCO}^{\mathrm{TM}}$ (Pulsion Medical Systems, Munich, Germany); in this case the calibration technique used was the transpulmonary thermodilution (TPTD). The uncalibrated SV $\left(\mathrm{SV}_{\text {PCUNCAL }}\right)$ was measured via the third-generation Flotrac/Vigileo ${ }^{\mathrm{TM}}$ monitor (Edwards Lifesciences, Irvine, CA, USA). The lowest limits of agreement in terms of percentage error (PE) were found for TPTD both in aortic stenosis (AS) and aortic insufficiency (AI). In AS, TPTD and $\mathrm{SV}_{\text {PCCAL }}$ performed with an acceptable PE (26.3 and $25.7 \%$, respectively) compared to $\mathrm{SV}_{\text {PCUNCAL }}(50.4 \%)$. In $\mathrm{AI}$, both $\mathrm{SV}_{\mathrm{PCCAL}}$ and $\mathrm{SV}_{\mathrm{PCUNCAL}}$ did not show acceptable PE (51.5 and $61.9 \%$, respectively), while TPTD maintained good PE even in this context (PE $26.2 \%)$. Looking at the polar plot for trending capacity, all three methods showed an acceptable performance, proving that in terms of trending even devices with a poor level of accuracy can provide useful information.

In this elegant study the authors have provided further confirmation regarding PP analysis:

- When PP analysis is used in severe valvular disease, especially for AI, an accurate snapshot requires calibration (and recalibration).

- Calibration with TPTD performs well even during valvular disease.

- Trending is partially maintained even when accuracy is not at its best.

- Continuity may be more important than accuracy.

- Physiological examination should be considered to be (at least) as important as the classic physical examination.

Disclosures Maurizio Cecconi: Edwards Lifesciences, LiDCO, Deltex, Applied Physiology, Masimo, Bmeye, Cheetah, Imacor (travel expenses, honoraria, advisory board, unrestricted educational grant, research material). Manu Malbrain is member of the medical advisory board of Pulsion Medical Systems, a monitoring company. 


\section{References}

1. Malbrain MLNG, Van Regenmortel N, Himpe D (2012) Meeting report of the first international fluid academy day. Part 2: results of the survey on the knowledge of hemodynamic monitoring and fluid responsiveness. Fluids $1: 15-26$

2. Cecconi M, Wilson J, Rhodes A (2006) Pulse pressure analysis. In: Vincent JL (ed ) Yearbook of intensive care and emergency medicine. Springer, Heidelberg, pp 176-184

3. Malbrain ML, De Potter P, Deeren D (2005) Cost-effectiveness of minimally invasive hemodynamic monitoring. Yearbook of intensive care and emergency medicine. Springer, Heidelberg, pp 603-618

4. Sagawa K, Lie RK, Schaefer J (1990) Translation of Otto Frank's paper "Die Grundform des Arteriellen Pulses" Zeitschrift fur Biologie 37:483-526 (1899). J Mol Cell Cardiol 22:253-254

5. Erlanger J, Hooker DR (1904) An experimental study of blood-pressure and of pulse-pressure in man. Johns Hopkins Hospital Reports 12:145-378

6. Wesseling KH, de Wit B, Weber JAP, Ty Smith N (1983) A simple device for the continuous measurement of cardiac output. Adv Cardiovasc Physiol 5:16-52

7. Alhashemi JA, Cecconi M, Hofer CK (2011) Cardiac output monitoring: an integrative perspective. Crit Care 15:214

8. Palmers P, Vidts W, Ameloot K, Cordemans C, Van Regenmortel N, De laet I, Schoonheydt K, Dits H, Eichhorn V, Reuter D, Malbrain MLNG (2012) Assessment of three minimally invasive continuous cardiac output measurement methods in critically ill patients and a review of the literature. Anestezjol Intens Ter 44 (in press)
9. Cecconi M, Rhodes A (2010) Pulse pressure analysis: to make a long story short. Crit Care 14:175

10. Cecconi M, Rhodes A, Poloniecki J, Della Rocca G, Grounds RM (2009) Bench-to-bedside review: the importance of the precision of the reference technique in method comparison studies - with specific reference to the measurement of cardiac output. Crit Care 13:201

11. Feldman JM (2009) Is it a bird? Is it a plane? The role of patient monitors in medical decision making. Anesth Analg 108:707-710

12. Broch O, Renner J, Gruenewald M, Meybohm P, Schottler J, Steinfath M, Malbrain M, Bein B (2012) A comparison of third-generation semiinvasive arterial waveform analysis with thermodilution in patients undergoing coronary surgery. Scientific World J 2012:451081

13. Critchley LA (2011) Pulse contour analysis: is it able to reliably detect changes in cardiac output in the haemodynamically unstable patient? Crit Care 15:106

14. Monnet X, Letierce A, Hamzaoui O, Chemla D, Anguel N, Osman D, Richard C, Teboul JL (2011) Arterial pressure allows monitoring the changes in cardiac output induced by volume expansion but not by norepinephrine. Crit Care Med 39:1394-1399

15. Sakka SG, Kozieras J, Thuemer O, van Hout N (2007) Measurement of cardiac output: a comparison between transpulmonary thermodilution and uncalibrated pulse contour analysis. $\mathrm{Br}$ J Anaesth 99:337-342
16. Cecconi M, Dawson D, Grounds RM, Rhodes A (2009) Lithium dilution cardiac output measurement in the critically ill patient: determination of precision of the technique. Intensive Care Med 35:498-504

17. Cecconi M, Fawcett J, Grounds RM, Rhodes A (2008) A prospective study to evaluate the accuracy of pulse power analysis to monitor cardiac output in critically ill patients. BMC Anesthesiol $8: 3$

18. Kavanagh BP, Meyer LJ (2005) Normalizing physiological variables in acute illness: five reasons for caution. Intensive Care Med 31:1161-1167

19. Sakka SG, Reuter DA, Perel A (2012) The transpulmonary thermodilution technique. J Clin Monit Comput 26:347-353

20. Petzoldt M, Riedel C, Braeunig J, Haas S, Goepfert MS, Treede H, Baldus S, Goetz AE, Reuter DA (2012) Stroke volume determination using transcardiopulmonary thermodilution and arterial pulse contour analysis in severe aortic valve disease. Intensive Care Med. doi: 10.1007/s00134-012-2786-7 